\title{
Special Solutions and Linear Monodromy for the Two-Dimensional Degenerate Garnier System G(1112)
}

Kazuo KANEKO

Seki Kowa Institute of Mathematics, Yokkaichi University,

Kayaucho, Yokkaichi, Mie, 512-8512, Japan

E-mail: dr_kaneko_k@yahoo.co.jp

Received October 24, 2013, in final form June 14, 2014; Published online July 05, 2014

http://dx.doi.org/10.3842/SIGMA.2014.069

\begin{abstract}
We have classified special solutions around the origin for the two-dimensional degenerate Garnier system G(1112) with generic values of complex parameters, whose linear monodromy can be calculated explicitly.
\end{abstract}

Key words: two-dimensional degenerate Garnier system; monodromy data

2010 Mathematics Subject Classification: 34M55; 33C15

\section{Introduction}

We have studied special solutions with generic values of complex parameters for the fourth, fifth, sixth and third Painlevé equations, for which the monodromy data of the associated linear equation (we call linear monodromy) can be calculated explicitly [9, 11, 10, 12]. These papers are based on A.V. Kitaev's idea who calculated first the linear monodromy with generic value of complex parameter explicitly by taking examples of the first and second Painlevé equations [14]. We remark that P. Appell [1] also studied the symmetric solutions to the first and second Painlevé equations, but he did not study linear monodromy problems.

The Garnier system was derived by R. Garnier (1912) as the extension of the sixth Painlevé equation [4]. The original Garnier system has $n$ variables and is expressed in the nonlinear partial differential equations system, whose dimension of the solution space is $2 n$. There are few research for the special solutions to the Garnier system compared with Painlevé equations. We will study the Garnier transcendents by applying first the same method to the two-dimensional Garnier system, which we have used for the Painlevé equations above. Some new discovery is expected by viewing Painlevé equations from the Garnier system.

Two-dimensional Garnier system has the following degeneration diagram similar to the Painlevé equations [13]:

$$
\begin{aligned}
& \mathrm{G}(11111) \rightarrow \mathrm{G}(1112) \rightarrow \mathrm{G}(122) \rightarrow \mathrm{G}(23)
\end{aligned}
$$

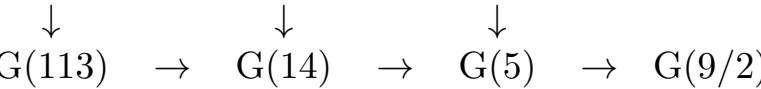

(The degeneration from $\mathrm{G}(113)$ to $\mathrm{G}(23)$ also exists.) Numbers in brackets represents a partition of 5 . The number 1 represents the regular singular point and the number $r+1$ represents an irregular singular point of Poincaré rank $r$. The two-dimensional Garnier system G(11111) which is the extension of the sixth Painlevé equation $\mathrm{P}_{\mathrm{VI}}$ degenerates step by step to the twodimensional degenerate Garnier system $\mathrm{G}(9 / 2)$ which is the extension of the first Painlevé equation $\mathrm{P}_{\mathrm{I}}$. 
The purpose of this paper is to obtain the special solutions to the system $G(1112)$, for which the linear monodromy $\left\{M_{0}, M_{1}=S_{1}^{(1)} S_{2}^{(1)} e^{2 \pi i T_{1}}, M_{t_{2}}, M_{\infty}\right\}$ can be calculated explicitly.

The two-dimensional degenerate Garnier system $\mathrm{G}(1112)\left\{K_{1}, K_{2}, \lambda_{1}, \lambda_{2}, \mu_{1}, \mu_{2}, t_{1}, t_{2}\right\}$ is derived as the extension of the fifth Painlevé equation by the isomonodromic deformation of the second kind, non-Fuchsian ordinary differential equation, which has three regular singularities and one irregular singularity of Poincaré rank 1 on the Riemann sphere $[13,16]$ :

$$
\begin{aligned}
\frac{d^{2} \psi}{d x^{2}}+ & {\left[\frac{1-\alpha_{0}}{x}+\frac{\eta t_{1}}{(x-1)^{2}}+\frac{2-\alpha_{1}}{x-1}+\frac{1-\alpha_{2}}{x-t_{2}}-\frac{1}{x-\lambda_{1}}-\frac{1}{x-\lambda_{2}}\right] \frac{d \psi}{d x} } \\
+ & {\left[\frac{\nu\left(\nu+\alpha_{\infty}\right)}{x(x-1)}-\frac{t_{1} K_{1}}{x(x-1)^{2}}-\frac{t_{2}\left(t_{2}-1\right) K_{2}}{x(x-1)\left(x-t_{2}\right)}\right.} \\
& \left.+\frac{\lambda_{1}\left(\lambda_{1}-1\right) \mu_{1}}{x(x-1)\left(x-\lambda_{1}\right)}+\frac{\lambda_{2}\left(\lambda_{2}-1\right) \mu_{2}}{x(x-1)\left(x-\lambda_{2}\right)}\right] \psi=0,
\end{aligned}
$$

where $K_{1}$ and $K_{2}$ are Hamiltonians, $\lambda_{1}, \lambda_{2}, \mu_{1}$ and $\mu_{2}$ are the Garnier functions, $t_{1}$ and $t_{2}$ are deformation parameters and $\alpha_{j}(j=0,1,2, \infty), \nu \in \mathbb{C}$ and $\eta \in \mathbb{C}^{\times}$are complex parameters. The Riemann scheme of (1.1) is

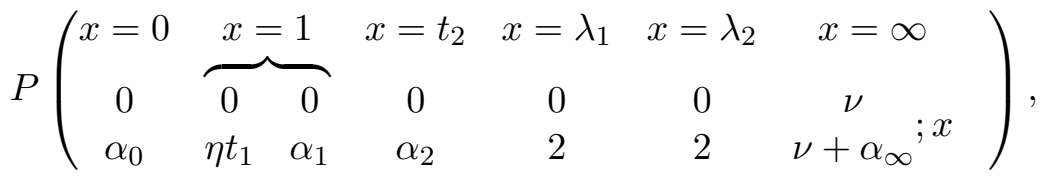

$$
\begin{aligned}
& \alpha_{0}+\alpha_{1}+\alpha_{2}+\alpha_{\infty}=1-2 \nu \text {. }
\end{aligned}
$$

This is also derived by the confluence of two regular singularities $x=t_{1}$ and $x=1$ in the two-dimensional Garnier system G(11111).

G(1112) has movable algebraic branch points and Hamiltonian structure expressed in rational function. We have the two-dimensional degenerate Garnier system $\mathcal{H}_{2}(1112)\left\{H_{1}, H_{2}, q_{1}, q_{2}\right.$, $\left.p_{1}, p_{2}, s_{1}, s_{2}\right\}$ by the canonical transformations:

$$
\begin{aligned}
& s_{1}=\frac{1}{t_{1}}, \quad s_{2}=\frac{t_{2}}{t_{2}-1}, \quad-t_{1}\left(t_{2}-1\right) q_{1}=\left(\lambda_{1}-1\right)\left(\lambda_{2}-1\right), \\
& \left(t_{2}-1\right)^{2} q_{2}=\left(\lambda_{1}-t_{2}\right)\left(\lambda_{2}-t_{2}\right), \quad \mu_{i}=\frac{q_{1} p_{1}}{\lambda_{i}-1}+\frac{q_{2} p_{2}}{\lambda_{i}-t_{2}}, \quad i=1,2, \\
& H_{1}=-t_{1}^{2}\left(K_{1}+\sum_{j=1}^{2} p_{j} \frac{\partial q_{j}}{\partial t_{1}}\right), \quad H_{2}=-\left(t_{2}-1\right)^{2}\left(K_{2}+\sum_{j=1}^{2} p_{j} \frac{\partial q_{j}}{\partial t_{2}}\right), \\
& \sum_{j=1}^{2}\left(d p_{j} \wedge d q_{j}-d H_{j} \wedge d s_{j}\right)=\sum_{j=1}^{2}\left(d \mu_{j} \wedge d \lambda_{j}-d K_{j} \wedge d t_{j}\right) .
\end{aligned}
$$

$\mathcal{H}_{2}$ has the Painlevé property and the polynomial Hamiltonian structure [7, 13, 16]. We obtain the special solutions in the Hamiltonian system $\mathcal{H}_{2}$ and then inversely transform them to the solutions in the Hamiltonian system G(1112), which are substituted into the linear equation (1.1). We obtain eight meromorphic solutions with generic values of complex parameters around the origin $\left(t_{1}, t_{2}\right)=(0,0)$, which we name the solutions $(1),(2), \ldots,(8)$.

The calculation of the linear monodromy consists of three steps. The first step is taking the limit $\left(t_{1}, t_{2}\right) \rightarrow(0,0)$ after substituting the solution into the linear equation (1.1). We call this step "the first limit", in which the linear monodromy matrices $M_{0}$ and $M_{t_{2}}$ are calculated as the confluent linear monodromy $M_{t_{2}} M_{0}$. In the second step, we separate this confluent linear monodromy $M_{t_{2}} M_{0}$. After transforming the linear equation (1.1) by putting $x=t_{2} \xi$ and substituting the solution into the linear equation (1.1), we take the limit $\left(t_{1}, t_{2}\right) \rightarrow(0,0)$. We 
call this step "the second limit". In the third step, we transform the linear equation (1.1) by putting $x-1=\eta t_{1} / z$ which keeps the irregularity at $x=1$ so that we can calculate the Stokes matrices $\left\{S_{1}^{(1)}, S_{2}^{(1)}\right\}$. We call this step "the third limit".

Each of the obtained eight meromorphic solutions with generic values of complex parameters around the origin $\left(t_{1}, t_{2}\right)=(0,0)$ has the remarkable characteristics, respectively. The four solutions make the two monodromy matrices commutable and the Stokes matrices around $x=1$ unity and the other four solutions make the three monodromy matrices commutable, which are summarized in Theorem 4.

In Appendix A, we show the fundamental solutions and the associated monodromy matrices of Gauss hypergeometric equation and Kummer's equation. In Appendix B, we show the BriotBouquet's theorem for a system of partial differential equations in two variables and short comment on it, how it proves convergence of the eight solutions.

\section{The two-dimensional degenerate Garnier system $\mathcal{H}_{2}(1112)$}

In this section, we write down the polynomial Hamiltonians $H_{1}, H_{2}$ and the Hamiltonian system $\mathcal{H}_{2}(1112)$.

- Hamiltonians $H_{1}$ and $H_{2}$ :

$$
\begin{aligned}
& s_{1}^{2} H_{1}= q_{1}^{2}\left(q_{1}-s_{1}\right) p_{1}^{2}+2 q_{1}^{2} q_{2} p_{1} p_{2}+q_{1} q_{2}\left(q_{2}-s_{2}\right) p_{2}^{2} \\
&-\left[\left(\alpha_{0}+\alpha_{2}-1\right) q_{1}^{2}+\alpha_{1} q_{1}\left(q_{1}-s_{1}\right)+\eta\left(q_{1}-s_{1}\right)+\eta s_{1} q_{2}\right] p_{1} \\
&-\left[\left(\alpha_{0}+\alpha_{1}-1\right) q_{1} q_{2}+\alpha_{2} q_{1}\left(q_{2}-s_{2}\right)-\eta\left(s_{2}-1\right) q_{2}\right] p_{2}+\nu\left(\nu+\alpha_{\infty}\right) q_{1}, \\
& s_{2}\left(s_{2}-1\right) H_{2}=q_{1}^{2} q_{2} p_{1}^{2}+2 q_{1} q_{2}\left(q_{2}-s_{2}\right) p_{1} p_{2} \\
&+\left[q_{2}\left(q_{2}-1\right)\left(q_{2}-s_{2}\right)+\frac{s_{2}\left(s_{2}-1\right)}{s_{1}} q_{1} q_{2}\right] p_{2}^{2} \\
&-\left[\left(\alpha_{0}+\alpha_{1}-1\right) q_{1} q_{2}+\alpha_{2} q_{1}\left(q_{2}-s_{2}\right)-\eta\left(s_{2}-1\right) q_{2}\right] p_{1} \\
&-\left[\left(\alpha_{0}-1\right) q_{2}\left(q_{2}-1\right)+\alpha_{1} q_{2}\left(q_{2}-s_{2}\right)+\alpha_{2}\left(q_{2}-1\right)\left(q_{2}-s_{2}\right)\right. \\
&\left.+\frac{s_{2}\left(s_{2}-1\right)}{s_{1}}\left(\alpha_{2} q_{1}+\eta q_{2}\right)\right] p_{2}+\nu\left(\nu+\alpha_{\infty}\right) q_{2} .
\end{aligned}
$$

- Hamiltonian system $\mathcal{H}_{2}(1112)$ :

$$
\begin{aligned}
& -t_{1} \frac{\partial q_{1}}{\partial t_{1}}=s_{1} \frac{\partial H_{1}}{\partial p_{1}}, \quad-t_{1} \frac{\partial q_{2}}{\partial t_{1}}=s_{1} \frac{\partial H_{1}}{\partial p_{2}}, \quad t_{1} \frac{\partial p_{1}}{\partial t_{1}}=s_{1} \frac{\partial H_{1}}{\partial q_{1}}, \quad t_{1} \frac{\partial p_{2}}{\partial t_{1}}=s_{1} \frac{\partial H_{1}}{\partial p_{1}}, \\
& \frac{\partial q_{1}}{\partial s_{2}}=\frac{\partial H_{2}}{\partial p_{1}}, \quad \frac{\partial q_{2}}{\partial s_{2}}=\frac{\partial H_{2}}{\partial p_{2}}, \quad-\frac{\partial p_{1}}{\partial s_{2}}=\frac{\partial H_{2}}{\partial q_{1}}, \quad-\frac{\partial p_{2}}{\partial s_{2}}=\frac{\partial H_{2}}{\partial q_{2}} .
\end{aligned}
$$

Remark 1. We use $t_{1}\left(=1 / s_{1}\right)$ instead of $s_{1}$ to apply the Briot-Bouquet's theorem [3] at the origin.

\section{Meromorphic solutions around the origin $\left(t_{1}=1 / s_{1}, s_{2}\right)=(0,0)$}

In this section, we give the calculated meromorphic solutions around $\left(t_{1}=1 / s_{1}, s_{2}\right)=(0,0)$, which are satisfied with the Hamiltonian system $\mathcal{H}_{2}(1112)$. 
When $q_{i}$ and $p_{i}(i=1,2)$ are meromorphic, they have at most a simple pole around $\left(t_{1}=\right.$ $\left.1 / s_{1}, s_{2}\right)=(0,0)$. Let $R=\mathbb{C}\left\{\left\{t_{1}, s_{2}\right\}\right\}$ be the ring of convergent power series of $t_{1}\left(=1 / s_{1}\right)$ and $s_{2}$ around the origin and for $u_{1}, u_{2}, \ldots, u_{n} \in R$, let $\left\langle u_{1}, u_{2}, \ldots, u_{n}\right\rangle$ be the ideal of $R$ generated by $u_{1}, u_{2}, \ldots, u_{n}$.

Theorem 1. For generic values of the complex parameters $\left\{\alpha_{0}, \alpha_{1}, \alpha_{2}, \alpha_{\infty}, \nu, \eta\right\}$, the Hamiltonian system $\mathcal{H}_{2}(1112)$ has the following eight meromorphic solutions around $\left(t_{1}=1 / s_{1}, s_{2}\right)=$ $(0,0)$ :

$$
\begin{aligned}
& q_{1}=\frac{\eta}{\alpha_{\infty}}+\left\langle t_{1}, s_{2}\right\rangle, \quad q_{2}=\frac{\alpha_{\infty}+\alpha_{1}}{\alpha_{\infty}}+\left\langle t_{1}, s_{2}\right\rangle \\
& p_{1}=\frac{\left(\alpha_{\infty}+\alpha_{1}\right)\left(\nu+\alpha_{2}\right)}{\alpha_{\infty}\left(1-\alpha_{\infty}-\alpha_{1}\right)} t_{1} s_{2}+\left\langle t_{1}^{3}, t_{1}^{2} s_{2}, s_{2}^{2}\right\rangle, \quad p_{2}=\frac{-\nu \alpha_{\infty}}{\alpha_{\infty}+\alpha_{1}}+\left\langle t_{1}, s_{2}\right\rangle,
\end{aligned}
$$

$$
\begin{aligned}
& q_{1}=\frac{-\eta}{\alpha_{\infty}}+\left\langle t_{1}, s_{2}\right\rangle, \quad q_{2}=\frac{\alpha_{\infty}-\alpha_{1}}{\alpha_{\infty}}+\left\langle t_{1}, s_{2}\right\rangle, \\
& p_{1}=\frac{\left(\alpha_{\infty}-\alpha_{1}\right)\left(\nu+\alpha_{2}+\alpha_{\infty}\right)}{\alpha_{\infty}\left(1+\alpha_{\infty}-\alpha_{1}\right)} t_{1} s_{2}+\left\langle t_{1}^{3}, t_{1}^{2} s_{2}, s_{2}^{2}\right\rangle, \quad p_{2}=\frac{-\left(\alpha_{\infty}+\nu\right)}{\alpha_{\infty}-\alpha_{1}} \alpha_{\infty}+\left\langle t_{1}, s_{2}\right\rangle,
\end{aligned}
$$

$$
\begin{aligned}
& q_{1}=\frac{-\eta}{\alpha_{1}}+\left\langle t_{1}, s_{2}\right\rangle, \quad q_{2}=s_{2}\left(\frac{\alpha_{2}}{\alpha_{0}+\alpha_{2}}+\left\langle t_{1}, s_{2}\right\rangle\right), \\
& p_{1}=\frac{\nu\left(\nu+\alpha_{\infty}\right)}{1-\alpha_{1}} t_{1}+\left\langle s_{2}, t_{1}^{2}\right\rangle, \quad p_{2}=\frac{1}{s_{2}}\left(\frac{\nu\left(\nu+\alpha_{\infty}\right)}{1-\alpha_{0}-\alpha_{2}} s_{2}+\left\langle t_{1}^{2}, t_{1} s_{2}, s_{2}^{2}\right\rangle\right),
\end{aligned}
$$

(4) $q_{1}=\frac{-\eta}{\alpha_{1}}+\left\langle t_{1}, s_{2}\right\rangle, \quad q_{2}=s_{2}\left(\frac{\alpha_{2}}{\alpha_{2}-\alpha_{0}}+\left\langle t_{1}, s_{2}\right\rangle\right)$,

$$
p_{1}=\frac{\left(\nu+\alpha_{2}\right)\left(\nu+\alpha_{2}+\alpha_{\infty}\right)}{1-\alpha_{1}} t_{1}+\left\langle s_{2}, t_{1}^{2}\right\rangle, \quad p_{2}=\frac{1}{s_{2}}\left(\alpha_{0}-\alpha_{2}+\left\langle t_{1}, s_{2}\right\rangle\right),
$$

$$
\begin{array}{ll}
q_{1}=\frac{1}{t_{1}}\left(\frac{\alpha_{\infty}+\alpha_{0}+\alpha_{2}}{\alpha_{\infty}}+\left\langle t_{1}, s_{2}\right\rangle\right), & q_{2}=\frac{-\alpha_{2}}{\alpha_{\infty}} s_{2}+\left\langle t_{1}^{2}, t_{1} s_{2}, s_{2}^{2}\right\rangle, \\
p_{1}=t_{1}\left(\frac{-\nu \alpha_{\infty}}{\alpha_{\infty}+\alpha_{0}+\alpha_{2}}+\left\langle t_{1}, s_{2}\right\rangle\right), & p_{2}=\frac{\eta \nu \alpha_{\infty} t_{1}}{\left(\alpha_{1}+2 \nu\right)\left(\alpha_{\infty}+\alpha_{0}+\alpha_{2}\right)}+\left\langle s_{2}, t_{1}^{2}\right\rangle,
\end{array}
$$

(6) $q_{1}=\frac{1}{t_{1}}\left(\frac{\alpha_{\infty}-\alpha_{0}-\alpha_{2}}{\alpha_{\infty}}+\left\langle t_{1}, s_{2}\right\rangle\right), \quad q_{2}=\frac{\alpha_{2}}{\alpha_{\infty}} s_{2}+\left\langle t_{1}^{2}, t_{1} s_{2}, s_{2}^{2}\right\rangle$,

$$
p_{1}=t_{1}\left(\frac{-\left(\nu+\alpha_{\infty}\right) \alpha_{\infty}}{\alpha_{\infty}-\alpha_{0}-\alpha_{2}}+\left\langle t_{1}, s_{2}\right\rangle\right)
$$

$$
\begin{aligned}
& p_{2}=\frac{\eta \alpha_{\infty}\left(\nu+\alpha_{\infty}\right) t_{1}}{\left(\alpha_{\infty}-\alpha_{0}-\alpha_{2}\right)\left(1-\alpha_{\infty}+\alpha_{0}+\alpha_{2}\right)}+\left\langle s_{2}, t_{1}^{2}\right\rangle, \\
& q_{1}=\frac{1}{t_{1}}\left(\frac{\alpha_{\infty}+\alpha_{0}-\alpha_{2}}{\alpha_{\infty}}+\left\langle t_{1}, s_{2}\right\rangle\right), \quad q_{2}=s_{2}\left(\frac{\alpha_{2}}{\alpha_{\infty}}+\left\langle t_{1}, s_{2}\right\rangle\right),
\end{aligned}
$$$$
p_{1}=t_{1}\left(\frac{-\left(\nu+\alpha_{2}\right) \alpha_{\infty}}{\alpha_{\infty}+\alpha_{0}-\alpha_{2}}+\left\langle t_{1}, s_{2}\right\rangle\right), \quad p_{2}=\frac{1}{s_{2}}\left(\alpha_{\infty}+\left\langle t_{1}, s_{2}\right\rangle\right),
$$

$$
\begin{aligned}
& q_{1}=\frac{1}{t_{1}}\left(\frac{\alpha_{\infty}-\alpha_{0}+\alpha_{2}}{\alpha_{\infty}}+\left\langle t_{1}, s_{2}\right\rangle\right), \quad q_{2}=s_{2}\left(\frac{-\alpha_{2}}{\alpha_{\infty}}+\left\langle t_{1}, s_{2}\right\rangle\right), \\
& p_{1}=t_{1}\left(\frac{-\left(\nu+\alpha_{2}+\alpha_{\infty}\right) \alpha_{\infty}}{\alpha_{\infty}-\alpha_{0}+\alpha_{2}}+\left\langle t_{1}, s_{2}\right\rangle\right), \quad p_{2}=\frac{1}{s_{2}}\left(-\alpha_{\infty}+\left\langle t_{1}, s_{2}\right\rangle\right) .
\end{aligned}
$$

\section{Remark 2.}

1. Higher order expansions of these solutions are uniquely determined recursively by the Hamiltonian system and do not contain any other parameter than the complex parameters $\left\{\alpha_{0}, \alpha_{1}, \alpha_{2}, \alpha_{\infty}, \nu, \eta\right\}$. 
2. These solutions are convergent by Briot-Bouquet's theorem (see Appendix B).

3. The values of complex parameters are generic and should be excluded the values with which the denominator of the coefficients become zero in the solutions above, that is, $\left\{\alpha_{1}, \alpha_{\infty}, \alpha_{1} \pm \alpha_{\infty}, \alpha_{0} \pm \alpha_{2}, \alpha_{0} \pm \alpha_{2} \pm \alpha_{\infty}\right\} \notin \mathbb{Z}$.

\section{The linear monodromy}

In this section, we calculate the linear monodromy for the solutions (1) and (5).

\subsection{For the solution (1)}

\subsubsection{The first limit}

After substituting the solution (1) into the linear equation (1.1), we take the limit $\left(t_{1}, t_{2}\right) \rightarrow$ $(0,0)$. Hereafter we call this as the first limit. Then the linear equation (1.1) becomes

$$
\begin{aligned}
\frac{d^{2} \psi_{1}}{d x^{2}} & +\left(\frac{2-\alpha_{0}-\alpha_{2}}{x}+\frac{1-\alpha_{1}}{x-1}-\frac{1}{x-b_{0}}\right) \frac{d \psi_{1}}{d x} \\
& +\left[\frac{\nu\left(\nu+\alpha_{\infty}\right)}{x(x-1)}-k_{2}\left(\frac{1}{x(x-1)}-\frac{1}{x^{2}}\right)+\frac{m_{2}}{x(x-1)\left(x-b_{0}\right)}\right] \psi_{1}=0,
\end{aligned}
$$

where $b_{0}=\frac{\alpha_{\infty}+\alpha_{1}}{\alpha_{\infty}}, k_{2}=\nu\left(1-\alpha_{0}-\alpha_{2}-\nu\right)$ and $m_{2}=\frac{-\nu \alpha_{1}}{\alpha_{\infty}}$. This is a Heun's type equation with the Riemann scheme

$$
P\left(\begin{array}{ccccc}
x=0 \cdot t_{2} & x=1 & x=b_{0} & x=\infty & \\
-\nu & 0 & 0 & \nu & ; x \\
-1+\alpha_{0}+\alpha_{2}+\nu & \alpha_{1} & 2 & \nu+\alpha_{\infty} &
\end{array}\right) .
$$

The general solution of (4.1) is

$$
\psi_{1}=c_{1} x^{-\nu}+c_{2} x^{-1+\alpha_{0}+\alpha_{2}+\nu}(x-1)^{\alpha_{1}}, \quad c_{1}, c_{2} \in \mathbb{C} .
$$

By taking the first limit, two regular singular points become confluent as a regular singular point $[2,8]$. The linear monodromy around $x=0 \cdot t_{2}$ is obtained as a confluent one $\widetilde{M}_{t_{2}} \widetilde{M}_{0}$.

The linear monodromy $\left\{\widetilde{M}_{t_{2}} \widetilde{M}_{0}, \widetilde{M}_{1}, \widetilde{M}_{\infty}\right\}$ of (4.1) is

$$
\begin{aligned}
& \widetilde{M}_{t_{2}} \widetilde{M}_{0}=\left(\begin{array}{cc}
e^{-2 \pi i \nu} & 0 \\
0 & e^{2 \pi i\left(\alpha_{0}+\alpha_{2}+\nu\right)}
\end{array}\right), \quad \widetilde{M}_{1}=\left(\begin{array}{cc}
1 & 0 \\
0 & e^{2 \pi i \alpha_{1}}
\end{array}\right), \\
& \widetilde{M}_{\infty}=\left(\begin{array}{cc}
e^{2 \pi i \nu} & 0 \\
0 & e^{2 \pi i\left(\nu+\alpha_{\infty}\right)}
\end{array}\right), \quad \widetilde{M}_{\infty} \widetilde{M}_{1} \widetilde{M}_{t_{2}} \widetilde{M}_{0}=I_{2} .
\end{aligned}
$$

We should separate the confluent linear monodromy $\widetilde{M}_{t_{2}} \widetilde{M}_{0}$.

\subsubsection{The second limit}

In this section, we separate the confluent linear monodromy $\widetilde{M}_{t_{2}} \widetilde{M}_{0}$. After transforming the linear equation (1.1) with $x=t_{2} \xi$ and substituting the solution (1) into (1.1), we take the limit $\left(t_{1}, t_{2}\right) \rightarrow(0,0)$. Hereafter we call this as the second limit. By taking the second limit, $x=0$ and $x=t_{2}$ are separated and $x=1$ and $x=\infty$ become confluent (see Remark 4). Then $\psi_{2}(\xi)=\psi\left(t_{2} \xi, t_{1}, t_{2}\right)$ satisfies the following Gauss hypergeometric equation after taking the limit $\left(t_{1}, t_{2}\right) \longrightarrow(0,0)$

$$
\frac{d^{2} \psi_{2}}{d \xi^{2}}+\left(\frac{1-\alpha_{0}}{\xi}+\frac{1-\alpha_{2}}{\xi-1}\right) \frac{d \psi_{2}}{d \xi}+\frac{k_{2}}{\xi(\xi-1)} \psi_{2}=0
$$


with the Riemann scheme

$$
P\left(\begin{array}{cccc}
(x=0) & \left(x=t_{2}\right) & (x=1 \cdot \infty) & \\
\xi=0 & \xi=1 & \xi=\infty & \\
0 & 0 & \nu & ; \xi \\
\alpha_{0} & \alpha_{2} & 1-\alpha_{0}-\alpha_{2}-\nu &
\end{array}\right) .
$$

The linear monodromy $\left\{M_{0}, M_{t_{2}}, M_{\infty} M_{1}\right\}$ of (4.2) is

$$
\begin{aligned}
& M_{0}=\left(\begin{array}{cc}
1 & 0 \\
0 & e^{2 \pi i \alpha_{0}}
\end{array}\right), \quad M_{t_{2}}=C_{01}^{-1}\left(\begin{array}{cc}
1 & 0 \\
0 & e^{2 \pi i \alpha_{2}}
\end{array}\right) C_{01}, \\
& M_{\infty} M_{1}=C_{0 \infty}^{-1}\left(\begin{array}{cc}
e^{2 \pi i \nu} & 0 \\
0 & e^{2 \pi i\left(\alpha_{1}+\nu+\alpha_{\infty}\right)}
\end{array}\right) C_{0 \infty}, \quad M_{\infty} M_{1} M_{t_{2}} M_{0}=I_{2},
\end{aligned}
$$

where $C_{01}$ and $C_{0 \infty}$ are the connection matrices of the Gauss hypergeometric function (see Appendix A, Lemma 1). The linear monodromy $\left\{M_{0}, M_{t_{2}}, M_{\infty} M_{1}\right\}$ of (4.2) is equivalent to $\left\{\widetilde{M}_{t_{2}} \widetilde{M}_{0}, \widetilde{M}_{1}, \widetilde{M}_{\infty}\right\}$ of (4.1). We have

$$
M_{1}=P^{-1} \widetilde{M}_{1} P, \quad M_{\infty}=P^{-1} \widetilde{M}_{\infty} P, \quad M_{\infty} M_{1}=P^{-1} \widetilde{M}_{\infty} \widetilde{M}_{1} P
$$

for a matrix $P \in \mathrm{GL}(2, \mathbb{C})$. Therefore, $P C_{0 \infty}^{-1} \in \mathrm{GL}(2, \mathbb{C})$ is a diagonal matrix. We have

$$
M_{1}=C_{0 \infty}^{-1}\left(\begin{array}{cc}
1 & 0 \\
0 & e^{2 \pi i \alpha_{1}}
\end{array}\right) C_{0 \infty}, \quad M_{\infty}=C_{0 \infty}^{-1}\left(\begin{array}{cc}
e^{2 \pi i \nu} & 0 \\
0 & e^{2 \pi i\left(\nu+\alpha_{\infty}\right)}
\end{array}\right) C_{0 \infty} .
$$

Remark 3. The formal solution of the linear equation (1.1) around $x=1$ has the form

$$
\Psi^{(1)}=\left(I_{2}+\sum_{k=1}^{\infty} \widehat{\Psi}_{k}^{(1)}(x-1)^{k}\right)(x-1)^{T_{1}} e^{\frac{T}{x-1}},
$$

where $T=\operatorname{diag}\left(0, \eta t_{1}\right), T_{1}=\operatorname{diag}\left(0, \alpha_{1}\right), \widehat{\Psi}_{k}^{(1)}=\widehat{\Psi}_{k}^{(1)}\left(\lambda_{1}, \lambda_{2}, \mu_{1}, \mu_{2}, t_{1}, t_{2}, \alpha_{j}, \eta\right)$.

The series $\widehat{\Psi}^{(1)}=I_{2}+\sum_{k=1}^{\infty} \widehat{\Psi}_{k}^{(1)}(x-1)^{k}$ may be a divergent series since $x=1$ is an irregular singularity of the Poincaré rank one.

The Stokes regions $\widetilde{\mathcal{S}}_{j}$ around $x=1$ are given by

$$
\widetilde{\mathcal{S}}_{j}=\{x \in \mathbb{C}|-\varepsilon+(j-1) \pi<\arg (x-1)<j \pi+\varepsilon,| x-1 \mid<r\},
$$

where $\varepsilon$ and $r$ are sufficiently small. There exist holomorphic functions $\widetilde{\Psi}_{j}(x)$ of $(1.1)$ on $\widetilde{\mathcal{S}}_{j}$ such that

$$
\widetilde{\Psi}_{j}(x) \sim \widehat{\Psi}^{(1)} \quad \text { for } \quad x \rightarrow 1 \quad \text { and } \quad \Psi_{j}(x)=\widetilde{\Psi}_{j}(x)(x-1)^{T_{1}} e^{\frac{T}{x-1}}
$$

is a solution of $(1.1)$ on $\widetilde{\mathcal{S}}_{j}$. The Stokes matrix $S_{j}$ is defined by

$$
\Psi_{j+1}=\Psi_{j} S_{j}
$$

We notice that $\Psi_{3}=\Psi_{1}\left(x e^{-2 \pi i}\right) e^{2 \pi i T_{1}}$.

First by taking a limit $t_{1} \rightarrow 0$ after substituting the solution (1) into the linear equation (1.1), $x=1$ of (1.1) becomes a regular singular point. Since the coefficients $\widehat{\Psi}_{k}^{(1)}$ are finite in the limit $t_{1} \rightarrow 0$, the formal solution $\Psi^{(1)}$ exists even in the limit $t_{1} \rightarrow 0$. Therefore,

$$
\left.\Psi^{(1)}\right|_{t_{1}=0}=\left(I_{2}+\sum_{k=1}^{\infty}\left[\widehat{\Psi}_{k}^{(1)}\right]_{t_{1}=0}(x-1)^{k}\right)(x-1)^{T_{1}}
$$

has a regular singularity at $x=1$. Thus the Stokes matrix $S_{j}$ become $I_{2}$ for $j=1,2$. Then taking a limit $t_{2} \rightarrow 0$ makes two regular singular points $x=1$ and $x=\infty$ confluent $[2,8]$. 


\subsubsection{The third limit}

In this section, we have Stokes matrices around the irregular singular point $x=1$ by the transformation of the linear equation (1.1), which keeps the irregularity at $x=1$. Put

$$
x-1=\frac{\eta t_{1}}{z},
$$

then $\psi_{3}(z)=\psi\left(\frac{\eta t_{1}}{z}+1, t_{1}, t_{2}\right)$ satisfies the following degenerate Kummer's equation after taking the limit $\left(t_{1}, t_{2}\right) \rightarrow(0,0)$,

$$
\frac{d^{2} \psi_{3}}{d z^{2}}+\left(\frac{1+\alpha_{1}}{z}-\frac{1}{z-\alpha_{1}}-1\right) \frac{d \psi_{3}}{d z}=0 .
$$

We have the general solution

$$
\psi_{3}=c_{3} e^{z} z^{-\alpha_{1}}+c_{4}, \quad c_{3}, c_{4} \in \mathbb{C} .
$$

This means the formal solution around the irregular singular point $x=1(z=\infty)$ becomes convergent and Stokes matrices around $x=1$ become $I_{2}$.

Therefore, we have the linear monodromy for the solution (1) explicitly.

Theorem 2. For the solution (1), the linear monodromy of (1.1) is

$$
\begin{aligned}
& M_{0}=\left(\begin{array}{cc}
1 & 0 \\
0 & e^{2 \pi i \alpha_{0}}
\end{array}\right), \quad M_{t_{2}}=C_{01}^{-1}\left(\begin{array}{cc}
1 & 0 \\
0 & e^{2 \pi i \alpha_{2}}
\end{array}\right) C_{01}, \\
& M_{1}=S_{1}^{(1)} S_{2}^{(1)} C_{0 \infty}^{-1}\left(\begin{array}{cc}
1 & 0 \\
0 & e^{2 \pi i \alpha_{1}}
\end{array}\right) C_{0 \infty}, \quad S_{1}^{(1)}=S_{2}^{(1)}=I_{2}, \\
& M_{\infty}=C_{0 \infty}^{-1}\left(\begin{array}{cc}
e^{2 \pi i \nu} & 0 \\
0 & e^{2 \pi i\left(\nu+\alpha_{\infty}\right)}
\end{array}\right) C_{0 \infty}, \\
& C_{01}=\left(\begin{array}{cc}
\frac{\Gamma\left(1-\alpha_{0}\right) \Gamma\left(\alpha_{2}\right)}{\Gamma\left(1-\alpha_{0}-\nu\right) \Gamma\left(\alpha_{2}+\nu\right)} & \frac{\Gamma\left(1+\alpha_{0}\right) \Gamma\left(\alpha_{2}\right)}{\Gamma(1-\nu) \Gamma\left(\alpha_{0}+\alpha_{2}+\nu\right)} \\
\frac{\Gamma\left(1-\alpha_{0}\right) \Gamma\left(-\alpha_{2}\right)}{\Gamma(\nu) \Gamma\left(1-\alpha_{0}-\alpha_{2}-\nu\right)} & \frac{\Gamma\left(1+\alpha_{0}\right) \Gamma\left(-\alpha_{2}\right)}{\Gamma\left(\alpha_{0}+\nu\right) \Gamma\left(1-\alpha_{2}-\nu\right)}
\end{array}\right), \\
& C_{0 \infty}=\left(\begin{array}{cc}
\frac{e^{\pi i \nu} \Gamma\left(1-\alpha_{0}\right) \Gamma\left(1-\alpha_{0}-\alpha_{2}-2 \nu\right)}{\Gamma\left(1-\alpha_{0}-\alpha_{2}-\nu\right) \Gamma\left(1-\alpha_{0}-\nu\right)} & \frac{e^{\pi i\left(\alpha_{0}+\nu\right)} \Gamma\left(1+\alpha_{0}\right) \Gamma\left(1-\alpha_{0}-\alpha_{2}-2 \nu\right)}{\Gamma(1-\nu) \Gamma\left(1-\alpha_{2}-\nu\right)} \\
\frac{e^{\pi i\left(1-\alpha_{0}-\alpha_{2}-\nu\right)} \Gamma\left(1-\alpha_{0}\right) \Gamma\left(\alpha_{0}+\alpha_{2}+2 \nu-1\right)}{\Gamma(\nu) \Gamma\left(\alpha_{2}+\nu\right)} & \frac{e^{\pi i\left(1-\alpha_{2}-\nu\right)} \Gamma\left(1+\alpha_{0}\right) \Gamma\left(\alpha_{0}+\alpha_{2}+2 \nu-1\right)}{\Gamma\left(\alpha_{0}+\alpha_{2}+\nu\right) \Gamma\left(\alpha_{0}+\nu\right)}
\end{array}\right), \\
& M_{\infty} M_{1} M_{t_{2}} M_{0}=I_{2},
\end{aligned}
$$

for which $\left[M_{1}, M_{\infty}\right]=0$ holds.

\subsection{For the solutions (2), (3) and (4)}

Also for the solutions (2), (3) and (4), we have the similar results, which are summarized as follows.

- For the solution (2):

$$
\begin{aligned}
& M_{0}=\left(\begin{array}{cc}
1 & 0 \\
0 & e^{2 \pi i \alpha_{0}}
\end{array}\right), \quad M_{t_{2}}=C_{01}^{(2)-1}\left(\begin{array}{cc}
1 & 0 \\
0 & e^{2 \pi i \alpha_{2}}
\end{array}\right) C_{01}^{(2)}, \\
& M_{1}=S_{1}^{(1)} S_{2}^{(1)} C_{0 \infty}^{(2)-1}\left(\begin{array}{cc}
e^{2 \pi i \alpha_{1}} & 0 \\
0 & 1
\end{array}\right) C_{0 \infty}^{(2)}, \quad S_{1}^{(1)}=S_{2}^{(1)}=I_{2}, \\
& M_{\infty}=C_{0 \infty}^{(2)-1}\left(\begin{array}{cc}
e^{2 \pi i \nu} & 0 \\
0 & e^{2 \pi i\left(\nu+\alpha_{\infty}\right)}
\end{array}\right) C_{0 \infty}^{(2)}, \quad C_{01}^{(2)}=C_{01}\left(\nu+\alpha_{1}, \nu+\alpha_{\infty}, 1-\alpha_{0}\right), \\
& C_{0 \infty}^{(2)}=C_{0 \infty}\left(\nu+\alpha_{1}, \nu+\alpha_{\infty}, 1-\alpha_{0}\right), \quad M_{\infty} M_{1} M_{t_{2}} M_{0}=I_{2},
\end{aligned}
$$

for which $\left[M_{1}, M_{\infty}\right]=0$ holds. 
- For the solution (3):

$$
\begin{aligned}
& M_{0}=\left(\begin{array}{cc}
1 & 0 \\
0 & e^{2 \pi i \alpha_{0}}
\end{array}\right), \quad M_{t_{2}}=\left(\begin{array}{cc}
1 & 0 \\
0 & e^{2 \pi i \alpha_{2}}
\end{array}\right), \\
& M_{1}=S_{1}^{(1)} S_{2}^{(1)} C_{01}^{(3)-1}\left(\begin{array}{cc}
1 & 0 \\
0 & e^{2 \pi i \alpha_{1}}
\end{array}\right) C_{01}^{(3)}, \quad S_{1}^{(1)}=S_{2}^{(1)}=I_{2}, \\
& M_{\infty}=C_{0 \infty}^{(3)-1}\left(\begin{array}{cc}
e^{2 \pi i \nu} & 0 \\
0 & e^{2 \pi i\left(\nu+\alpha_{\infty}\right)}
\end{array}\right) C_{0 \infty}^{(3)}, \quad C_{01}^{(3)}=C_{01}\left(\nu, \nu+\alpha_{\infty}, 1-\alpha_{0}-\alpha_{2}\right), \\
& C_{0 \infty}^{(3)}=C_{0 \infty}\left(\nu, \nu+\alpha_{\infty}, 1-\alpha_{0}-\alpha_{2}\right), \quad M_{\infty} M_{1} M_{t_{2}} M_{0}=I_{2},
\end{aligned}
$$

for which $\left[M_{0}, M_{t_{2}}\right]=0$ holds.

- For the solution (4):

$$
\begin{aligned}
& M_{0}=\left(\begin{array}{cc}
1 & 0 \\
0 & e^{2 \pi i \alpha_{0}}
\end{array}\right), \quad M_{t_{2}}=\left(\begin{array}{cc}
1 & 0 \\
0 & e^{2 \pi i \alpha_{2}}
\end{array}\right), \\
& M_{1}=S_{1}^{(1)} S_{2}^{(1)} C_{01}^{(4)-1}\left(\begin{array}{cc}
1 & 0 \\
0 & e^{2 \pi i \alpha_{1}}
\end{array}\right) C_{01}^{(4)}, \quad S_{1}^{(1)}=S_{2}^{(1)}=I_{2}, \\
& M_{\infty}=C_{0 \infty}^{(4)-1}\left(\begin{array}{cc}
e^{2 \pi i \nu} & 0 \\
0 & e^{2 \pi i\left(\nu+\alpha_{\infty}\right)}
\end{array}\right) C_{0 \infty}^{(4)}, \quad C_{01}^{(4)}=C_{01}\left(\nu, \nu+\alpha_{\infty}, 1-\alpha_{2}\right), \\
& C_{0 \infty}^{(4)}=C_{0 \infty}\left(\nu, \nu+\alpha_{\infty}, 1-\alpha_{2}\right), \quad M_{\infty} M_{1} M_{t_{2}} M_{0}=I_{2},
\end{aligned}
$$

for which $\left[M_{0}, M_{t_{2}}\right]=0$ holds.

\subsection{For the solution (5)}

In this section, we calculate the linear monodromy for the solution (5) by the similar way to Subsection 4.1.

\subsubsection{The first limit}

After substituting the solution (5) into the linear equation (1.1), we take the limit $\left(t_{1}, t_{2}\right) \rightarrow$ $(0,0)$. At first we take a limit $t_{1} \rightarrow 0$ keeping $t_{2}$ as a non-zero constant, then we take $t_{2} \rightarrow 0$. Then the linear equation (1.1) becomes

$$
\begin{aligned}
\frac{d^{2} \psi_{1}}{d x^{2}} & +\left(\frac{1-\alpha_{0}-\alpha_{2}}{x}+\frac{2-\alpha_{1}}{x-1}-\frac{1}{x-b_{0}}\right) \frac{d \psi_{1}}{d x} \\
& +\left[\frac{\nu\left(\nu+\alpha_{\infty}\right)}{x(x-1)}-\frac{k_{1}}{x(x-1)^{2}}+\frac{m_{1}}{x(x-1)\left(x-b_{0}\right)}\right] \psi_{1}=0
\end{aligned}
$$

where $b_{0}=\frac{\alpha_{0}+\alpha_{2}}{-\alpha_{\infty}}, k_{1}=\nu\left(\nu+\alpha_{1}-1\right)$ and $m_{1}=\frac{\nu\left(\alpha_{0}+\alpha_{2}\right)}{\alpha_{\infty}}$. This is a Heun's type equation with the Riemann scheme

$$
P\left(\begin{array}{ccccc}
x=0 \cdot t_{2} & x=1 & x=b_{0} & x=\infty & \\
0 & -\nu & 0 & \nu & \\
\alpha_{0}+\alpha_{2} & \nu+\alpha_{1}-1 & 2 & \nu+\alpha_{\infty} & ; x
\end{array}\right) .
$$

The general solution of (4.3) is

$$
\psi_{1}=c_{5}(x-1)^{-\nu}+c_{6} x^{\alpha_{0}+\alpha_{2}}(x-1)^{\nu+\alpha_{1}-1}, \quad c_{5}, c_{6} \in \mathbb{C} .
$$


The linear monodromy $\left\{\widetilde{M}_{t_{2}} \widetilde{M}_{0}, \widetilde{M}_{1}, \widetilde{M}_{\infty}\right\}$ of (4.3) is

$$
\begin{array}{ll}
\widetilde{M}_{t_{2}} \widetilde{M}_{0}=\left(\begin{array}{cc}
1 & 0 \\
0 & e^{2 \pi i\left(\alpha_{0}+\alpha_{2}\right)}
\end{array}\right), & \widetilde{M}_{1}=\left(\begin{array}{cc}
e^{-2 \pi i \nu} & 0 \\
0 & e^{2 \pi i\left(\nu+\alpha_{1}\right)}
\end{array}\right), \\
\widetilde{M}_{\infty}=\left(\begin{array}{cc}
e^{2 \pi i \nu} & 0 \\
0 & e^{2 \pi i\left(\nu+\alpha_{\infty}\right)}
\end{array}\right), & \widetilde{M}_{\infty} \widetilde{M}_{1} \widetilde{M}_{t_{2}} \widetilde{M}_{0}=I_{2} .
\end{array}
$$

We should separate the confluent linear monodromy $\widetilde{M}_{t_{2}} \widetilde{M}_{0}$.

\subsubsection{The second limit}

In this section, we separate the confluent linear monodromy $\widetilde{M}_{t_{2}} \widetilde{M}_{0}$. After transforming the linear equation (1.1) with $x=t_{2} \xi$ and substituting the solution (5) into (1.1), we take the limit $\left(t_{1}, t_{2}\right) \rightarrow(0,0)$ as the same as the first limit.

By taking the second limit, $x=0$ and $x=t_{2}$ are separated and $x=1$ and $x=\infty$ become confluent. Then $\psi_{2}(\xi)=\psi\left(t_{2} \xi, t_{1}, t_{2}\right)$ satisfies the following degenerate Heun's equation after taking the limit $\left(t_{1}, t_{2}\right) \rightarrow(0,0)$,

$$
\frac{d^{2} \psi_{2}}{d \xi^{2}}+\left(\frac{1-\alpha_{0}}{\xi}+\frac{1-\alpha_{2}}{\xi-1}-\frac{1}{\xi-\xi_{\lambda_{2}}}\right) \frac{d \psi_{2}}{d \xi}=0
$$

where $\xi_{\lambda_{2}}=\frac{\alpha_{0}}{\alpha_{0}+\alpha_{2}}$. We have the general solution

$$
\psi_{2}=c_{7}+c_{8} \xi^{\alpha_{0}}(\xi-1)^{\alpha_{2}}, \quad c_{7}, c_{8} \in \mathbb{C} .
$$

The linear monodromy $\left\{M_{0}, M_{t_{2}}, M_{\infty} M_{1}\right\}$ of (4.4) is

$$
\begin{aligned}
& M_{0}=\left(\begin{array}{cc}
1 & 0 \\
0 & e^{2 \pi i \alpha_{0}}
\end{array}\right), \quad M_{t_{2}}=\left(\begin{array}{cc}
1 & 0 \\
0 & e^{2 \pi i \alpha_{2}}
\end{array}\right), \quad M_{\infty} M_{1}=\left(\begin{array}{cc}
1 & 0 \\
0 & e^{-2 \pi i\left(\alpha_{0}+\alpha_{2}\right)}
\end{array}\right), \\
& M_{\infty} M_{1} M_{t_{2}} M_{0}=I_{2} .
\end{aligned}
$$

\subsubsection{The third limit}

In this section, we calculate the Stokes matrices around the irregular singular point $x=1$ by the transformation of the linear equation (1.1), which keeps the irregularity at $x=1$. Put

$$
x-1=\frac{\eta t_{1}}{z}, \quad \psi=\left(\eta^{-1} z\right)^{\nu} \psi_{3}\left(z, t_{1}, t_{2}\right),
$$

then $\psi_{3}\left(z, t_{1}, t_{2}\right)=\left(\eta^{-1} z\right)^{-\nu} \psi\left(\frac{\eta t_{1}}{z}+1, t_{1}, t_{2}\right)$ satisfies the following Kummer's confluent hypergeometric equation after taking the limit $\left(t_{1}, t_{2}\right) \longrightarrow(0,0)$,

$$
\frac{d^{2} \psi_{3}}{d z^{2}}+\left(\frac{2 \nu+\alpha_{1}}{z}-1\right) \frac{d \psi_{3}}{d z}-\frac{\nu}{z} \psi_{3}=0
$$

A system of the fundamental solutions is

$$
\left({ }_{1} F_{1}\left(\nu, 2 \nu+\alpha_{1} ; z\right), z^{1-2 \nu-\alpha_{1}}{ }_{1} F_{1}\left(1-\nu-\alpha_{1}, 2-2 \nu-\alpha_{1} ; z\right)\right) .
$$

We have the Stokes matrices $S_{1}^{(1)}$ and $S_{2}^{(1)}$ around the irregular singular point $x=1(z=\infty)$ (see Appendix A, Lemma 2).

Summarizing the calculations above, we have the following theorem: 
Theorem 3. For the solution (5), the linear monodromy of (1.1) is

$$
\begin{aligned}
& M_{0}=C^{(5)}\left(\begin{array}{cc}
1 & 0 \\
0 & e^{2 \pi i \alpha_{0}}
\end{array}\right) C^{(5)-1}, \quad M_{t_{2}}=C^{(5)}\left(\begin{array}{cc}
1 & 0 \\
0 & e^{2 \pi i \alpha_{2}}
\end{array}\right) C^{(5)-1}, \\
& M_{1}=S_{1}^{(1)} S_{2}^{(1)} e^{2 \pi i T_{1}}, \quad e^{2 \pi i T_{1}}=\left(\begin{array}{cc}
1 & 0 \\
0 & e^{2 \pi i \alpha_{1}}
\end{array}\right), \quad S_{1}^{(1)}=\left(\begin{array}{cc}
1 & 0 \\
\frac{-2 \pi i e^{\pi i \alpha_{1}}}{\Gamma(\nu) \Gamma\left(1-\nu-\alpha_{1}\right)} & 1
\end{array}\right), \\
& S_{2}^{(1)}=\left(\begin{array}{cc}
1 & \frac{-2 \pi i e^{-2 \pi i \alpha_{1}}}{\Gamma(1-\nu) \Gamma\left(\nu+\alpha_{1}\right)} \\
0 & 1
\end{array}\right), \quad M_{\infty}=C^{(5)}\left(\begin{array}{cc}
e^{2 \pi i \nu} & 0 \\
0 & e^{2 \pi i\left(\nu+\alpha_{\infty}\right)}
\end{array}\right) C^{(5)-1}, \\
& C^{(5)}=C\left(\nu, 2 \nu+\alpha_{1}\right)=\left(\begin{array}{cc}
\frac{\Gamma\left(2 \nu+\alpha_{1}\right)}{\Gamma\left(\nu+\alpha_{1}\right)} e^{\pi i \nu} & \frac{\Gamma\left(2-2 \nu-\alpha_{1}\right)}{\Gamma(1-\nu)} e^{\pi i\left(1-\nu-\alpha_{1}\right)} \\
\frac{\Gamma\left(2 \nu+\alpha_{1}\right)}{\Gamma(\nu)} & \frac{\Gamma\left(2-2 \nu-\alpha_{1}\right)}{\Gamma\left(1-\nu-\alpha_{1}\right)}
\end{array}\right), \\
& M_{\infty} M_{t_{2}} M_{0} S_{1}^{(1)} S_{2}^{(1)} e^{2 \pi i T_{1}}=I_{2},
\end{aligned}
$$

for which $\left[M_{0}, M_{t_{2}}\right]=0,\left[M_{0}, M_{\infty}\right]=0$ and $\left[M_{t_{2}}, M_{\infty}\right]=0$ hold and $C^{(5)}$ is the connection matrix of Kummer's confluent hypergeometric function (see Appendix A, Lemma 2).

Remark 4. If we take a limit $\left(t_{1}, t_{2}\right) \rightarrow(0,0)$ along a curve $s_{2} \sim A t_{1}^{2}\left(A \in \mathbb{C}^{\times}\right)$, the limit of the last term in (1.1)

$$
\frac{\lambda_{2}\left(\lambda_{2}-1\right) \mu_{2}}{x(x-1)\left(x-\lambda_{2}\right)}
$$

is not zero. In our calculation, we take a special path from $\left(t_{1}, t_{2}\right)$ to $(0,0)$, such that the numerator of the above term tends to zero. Therefore we obtain different limit equations when we choose different paths for the first and the second limit equations. It may be a contradiction. But the whole of linear monodromy is the same even though some limit equations are different, since we have the Riemann-Hilbert correspondence. In our case, the third limit is the same for any path from $\left(t_{1}, t_{2}\right)$ to $(0,0)$, which is the main part of the linear monodromy for the solution (5).

\subsection{For the solutions (6), (7) and (8)}

We can determine the linear monodromy for the other solutions (6), (7) and (8), which are summarized as follows.

- For the solution (6):

$$
\begin{aligned}
& M_{0}=C^{(6)}\left(\begin{array}{cc}
1 & 0 \\
0 & e^{2 \pi i \alpha_{0}}
\end{array}\right) C^{(6)-1}, \quad M_{t_{2}}=C^{(6)}\left(\begin{array}{cc}
1 & 0 \\
0 & e^{2 \pi i \alpha_{2}}
\end{array}\right) C^{(6)-1}, \\
& M_{\infty}=C^{(6)}\left(\begin{array}{cc}
\left.e^{2 \pi i\left(\nu+\alpha_{\infty}\right.}\right) & 0 \\
0 & e^{2 \pi i \nu}
\end{array}\right) C^{(6)-1}, \quad M_{1}=S_{1}^{(1)} S_{2}^{(1)} e^{2 \pi i T_{1}}, \\
& e^{2 \pi i T_{1}}=\left(\begin{array}{cc}
1 & 0 \\
0 & e^{2 \pi i \alpha_{1}}
\end{array}\right), \quad C^{(6)}=C\left(\nu+\alpha_{\infty}, 2 \nu+2 \alpha_{\infty}+\alpha_{1}\right), \\
& S_{1}^{(1)}=S_{1}^{(\infty)}\left(\nu+\alpha_{\infty}, 2 \nu+2 \alpha_{\infty}+\alpha_{1}\right), \quad S_{2}^{(1)}=S_{2}^{(\infty)}\left(\nu+\alpha_{\infty}, 2 \nu+2 \alpha_{\infty}+\alpha_{1}\right), \\
& M_{\infty} M_{t_{2}} M_{0} S_{1}^{(1)} S_{2}^{(1)} e^{2 \pi i T_{1}}=I_{2},
\end{aligned}
$$

for which $\left[M_{0}, M_{t_{2}}\right]=0,\left[M_{0}, M_{\infty}\right]=0$ and $\left[M_{t_{2}}, M_{\infty}\right]=0$ hold.

- For the solution (7):

$$
M_{0}=C^{(7)}\left(\begin{array}{cc}
1 & 0 \\
0 & e^{2 \pi i \alpha_{0}}
\end{array}\right) C^{(7)-1}, \quad M_{t_{2}}=C^{(7)}\left(\begin{array}{cc}
e^{2 \pi i \alpha_{2}} & 0 \\
0 & 1
\end{array}\right) C^{(7)-1},
$$




$$
\begin{aligned}
& M_{\infty}=C^{(7)}\left(\begin{array}{cc}
e^{2 \pi i \nu} & 0 \\
0 & e^{2 \pi i\left(\nu+\alpha_{\infty}\right)}
\end{array}\right) C^{(7)-1}, \quad M_{1}=S_{1}^{(1)} S_{2}^{(1)} e^{2 \pi i T_{1}}, \\
& e^{2 \pi i T_{1}}=\left(\begin{array}{cc}
1 & 0 \\
0 & e^{2 \pi i \alpha_{1}}
\end{array}\right), \quad C^{(7)}=C\left(\nu+\alpha_{2}, 2 \nu+2 \alpha_{2}+\alpha_{1}\right), \\
& S_{1}^{(1)}=S_{1}^{\infty}\left(\nu+\alpha_{2}, 2 \nu+2 \alpha_{2}+\alpha_{1}\right), \quad S_{2}^{(1)}=S_{2}^{\infty}\left(\nu+\alpha_{2}, 2 \nu+2 \alpha_{2}+\alpha_{1}\right), \\
& M_{\infty} M_{t_{2}} M_{0} S_{1}^{(1)} S_{2}^{(1)} e^{2 \pi i T_{1}}=I_{2},
\end{aligned}
$$

for which $\left[M_{0}, M_{t_{2}}\right]=0,\left[M_{0}, M_{\infty}\right]=0$ and $\left[M_{t_{2}}, M_{\infty}\right]=0$ hold.

- For the solution (8):

$$
\begin{aligned}
& M_{0}=C^{(8)}\left(\begin{array}{cc}
e^{2 \pi i \alpha_{0}} & 0 \\
0 & 1
\end{array}\right)=C^{(8)-1}, \quad M_{t_{2}}=C^{(8)}\left(\begin{array}{cc}
1 & 0 \\
0 & e^{2 \pi i \alpha_{2}}
\end{array}\right) C^{(8)-1}, \\
& M_{\infty}=C^{(8)}\left(\begin{array}{cc}
e^{2 \pi i \nu} & 0 \\
0 & e^{2 \pi i\left(\nu+\alpha_{\infty}\right)}
\end{array}\right) C^{(8)-1}, \quad M_{1}=S_{1}^{(1)} S_{2}^{(1)} e^{2 \pi i T_{1}}, \\
& e^{2 \pi i T_{1}}=\left(\begin{array}{cc}
1 & 0 \\
0 & e^{2 \pi i \alpha_{1}}
\end{array}\right), \quad C^{(8)}=C\left(\nu+\alpha_{2}+\alpha_{\infty}, 2 \nu+2 \alpha_{2}+2 \alpha_{\infty}+\alpha_{1}\right), \\
& S_{1}^{(1)}=S_{1}^{\infty}\left(\nu+\alpha_{2}+\alpha_{\infty}, 2 \nu+2 \alpha_{2}+2 \alpha_{\infty}+\alpha_{1}\right), \\
& S_{2}^{(1)}=S_{2}^{\infty}\left(\nu+\alpha_{2}+\alpha_{\infty}, 2 \nu+2 \alpha_{2}+2 \alpha_{\infty}+\alpha_{1}\right), \\
& M_{\infty} M_{t_{2}} M_{0} S_{1}^{(1)} S_{2}^{(1)} e^{2 \pi i T_{1}}=I_{2},
\end{aligned}
$$

for which $\left[M_{0}, M_{\infty}\right]=0,\left[M_{0}, M_{t_{2}}\right]=0$ and $\left[M_{t_{2}}, M_{\infty}\right]=0$ hold.

Summarizing the all calculations above, we have the following theorem:

Theorem 4. The eight meromorphic solutions around the origin of the two-dimensional degenerate Garnier system $\mathrm{G}_{2}(1112)$ have the following characteristics:

- For the solution (1) and (2), $\left[M_{1}, M_{\infty}\right]=0$ and $S_{1}^{(1)}=S_{2}^{(1)}=I_{2}$ hold.

- For the solution (3) and (4), $\left[M_{0}, M_{t_{2}}\right]=0$ and $S_{1}^{(1)}=S_{2}^{(1)}=I_{2}$ hold.

- For the solution (5), (6), (7) and (8), $\left[M_{0}, M_{\infty}\right]=0,\left[M_{0}, M_{t_{2}}\right]=0$ and $\left[M_{t_{2}}, M_{\infty}\right]=0$ hold.

Remark 5. For the linear monodromy data $\left\{M_{0}, M_{\infty}, S_{1}^{(1)}, S_{2}^{(1)}, e^{2 \pi i T_{1}}\right\}$ of the fifth Painlevé equation, there are three meromorphic solutions around the origin: two solutions such that $\left[M_{0}, M_{\infty}\right]=0$ and one solution such that $S_{1}^{(1)}=S_{2}^{(1)}=I_{2}[11]$.

\section{A Gauss hypergeometric equation and Kummer's equation}

In this appendix, we show the fundamental solutions and the associated monodromy matrices of Gauss hypergeometric equation [15] and Kummer's equation [6].

\section{A.1 Gauss hypergeometric equation}

The Gauss hypergeometric equation is

$$
x(1-x) \frac{d^{2} \psi}{d x^{2}}+(\gamma-(\alpha+\beta+1) x) \frac{d \psi}{d x}-\alpha \beta \psi=0 .
$$


The Riemann scheme of (A.1) is

$$
P\left(\begin{array}{cccc}
x=0 & x=1 & x=\infty & \\
0 & 0 & \alpha & ; x \\
1-\gamma & \gamma-\alpha-\beta & \beta &
\end{array}\right),
$$

We list fundamental systems of solutions for (A.1) around $x=0,1, \infty$.

- Around $x=0$ :

$$
\psi^{(0)}=\left({ }_{2} F_{1}(\alpha, \beta, \gamma ; x) \quad x^{1-\gamma} F_{2}(\alpha+1-\gamma, \beta+1-\gamma, 2-\gamma ; x)\right) .
$$

- Around $x=1$ :

$$
\begin{aligned}
& \psi^{(1)}=\left(\begin{array}{ll}
\psi_{1}^{(1)} & \psi_{2}^{(1)}
\end{array}\right), \\
& \psi_{1}^{(1)}={ }_{2} F_{1}(\alpha, \beta, \alpha+\beta-\gamma+1 ; 1-x), \\
& \psi_{2}^{(1)}=(1-x)^{\gamma-\alpha-\beta}{ }_{2} F_{1}(\gamma-\alpha, \gamma-\beta, \gamma+1-\alpha-\beta ; 1-x) .
\end{aligned}
$$

- Around $x=\infty$ :

$$
\begin{aligned}
& \psi^{(\infty)}=\left(\begin{array}{ll}
\psi_{1}^{(\infty)} & \psi_{2}^{(\infty)}
\end{array}\right), \\
& \psi_{1}^{(\infty)}=x^{-\alpha}{ }_{2} F_{1}\left(\alpha, \alpha-\gamma+1, \alpha+1-\beta ; x^{-1}\right), \\
& \psi_{2}^{(\infty)}=x^{-\beta}{ }_{2} F_{1}\left(\beta, \beta-\gamma+1, \beta+1-\alpha ; x^{-1}\right) .
\end{aligned}
$$

The associated monodromy matrices $M_{j}(j=0,1, \infty)$ are as follows

$$
\begin{aligned}
& M_{0}=\left(\begin{array}{cc}
1 & 0 \\
0 & e^{-2 \pi i \gamma}
\end{array}\right), \quad M_{1}=C_{01}(\alpha, \beta, \gamma)^{-1}\left(\begin{array}{cc}
1 & 0 \\
0 & e^{2 \pi i(\gamma-\alpha-\beta)}
\end{array}\right) C_{01}(\alpha, \beta, \gamma), \\
& M_{\infty}=C_{0 \infty}(\alpha, \beta, \gamma)^{-1}\left(\begin{array}{cc}
e^{2 \pi i \alpha} & 0 \\
0 & e^{2 \pi i \beta}
\end{array}\right) C_{0 \infty}(\alpha, \beta, \gamma), \quad M_{\infty} M_{1} M_{0}=I_{2},
\end{aligned}
$$

where $C_{01}(\alpha, \beta, \gamma)$ and $C_{0 \infty}(\alpha, \beta, \gamma)$ are connection matrices which are shown in the following lemma.

Lemma 1. The Gauss hypergeometric function which is the solution of (A.1) has the following connection matrices between fundamental solutions around two singularities:

$$
\psi^{(i)}=\psi^{(j)} C_{i j}(\alpha, \beta, \gamma), \quad i, j \in\{0,1, \infty\},
$$

where $\psi^{(\nu)}(\nu \in\{0,1, \infty\})$ is the fundamental solution around the singularity $\nu$ and $C_{i j}(\alpha, \beta, \gamma)$ are the connection matrices which are shown as follows

$$
\begin{aligned}
C_{01}(\alpha, \beta, \gamma)= & \left(\begin{array}{ll}
\frac{\Gamma(\gamma) \Gamma(\gamma-\alpha-\beta)}{\Gamma(\gamma-\alpha) \Gamma(\gamma-\beta)} & \frac{\Gamma(2-\gamma) \Gamma(\gamma-\alpha-\beta)}{\Gamma(1-\alpha) \Gamma(1-\beta)} \\
\frac{\Gamma(\gamma) \Gamma(\alpha+\beta-\gamma)}{\Gamma(\alpha) \Gamma(\beta)} & \frac{\Gamma(2-\gamma) \Gamma(\alpha+\beta-\gamma)}{\Gamma(1+\alpha-\gamma) \Gamma(1+\beta-\gamma)}
\end{array}\right), \\
C_{0 \infty}(\alpha, \beta, \gamma)= & \left(\begin{array}{ll}
\frac{e^{\alpha \pi i} \Gamma(\gamma) \Gamma(\beta-\alpha)}{\Gamma(\beta) \Gamma(\gamma-\alpha)} & \frac{e^{(\alpha-\gamma+1) \pi i} \Gamma(2-\gamma) \Gamma(\beta-\alpha)}{\Gamma(1-\alpha) \Gamma(1-\gamma+\beta)} \\
\frac{e^{\beta \pi i} \Gamma(\gamma) \Gamma(\alpha-\beta)}{\Gamma(\alpha) \Gamma(\gamma-\beta)} & \frac{e^{(\beta-\gamma+1) \pi i} \Gamma(2-\gamma) \Gamma(\alpha-\beta)}{\Gamma(1-\beta) \Gamma(1-\gamma+\alpha)}
\end{array}\right), \\
C_{\infty 1}(\alpha, \beta, \gamma)= & \left.\begin{array}{cc}
\frac{\Gamma(1+\alpha-\beta) \Gamma(\gamma-\alpha-\beta)}{\Gamma(\gamma-\beta) \Gamma(1-\beta)} & \frac{\Gamma(1+\beta-\alpha) \Gamma(\gamma-\alpha-\beta)}{\Gamma(\gamma-\alpha) \Gamma(1-\alpha)} \\
\frac{e^{(\gamma-\alpha-\beta) \pi i} \Gamma(1+\alpha-\beta) \Gamma(\alpha+\beta-\gamma)}{\Gamma(1+\alpha-\gamma) \Gamma(\alpha)} & \frac{e^{(\gamma-\alpha-\beta) \pi i} \Gamma(1+\beta-\alpha) \Gamma(\alpha+\beta-\gamma)}{\Gamma(1+\beta-\gamma) \Gamma(\beta)}
\end{array}\right) .
\end{aligned}
$$




\section{A.2 Kummer's equation}

The Kummer's equation is

$$
\frac{d^{2} \phi}{d x^{2}}+\left(\frac{\gamma}{x}-1\right) \frac{d \phi}{d x}-\frac{\alpha}{x} \phi=0
$$

The Riemann scheme of (A.2) is

$$
P\left(\begin{array}{ccc}
x=0 & \overbrace{0}^{x=\infty} & \alpha \\
0 & \alpha
\end{array}\right) .
$$

Fundamental systems of solutions for (A.2) is given by

$$
\phi^{(0)}=\left({ }_{1} F_{1}(\alpha, \gamma ; x) \quad x^{1-\gamma} F_{1}(\alpha+1-\gamma, 2-\gamma ; x)\right) .
$$

Asymtotic solutions around $x=\infty$ is given by

$$
\begin{aligned}
& \phi_{1}^{(\infty)}\left(e^{-\pi i} x\right) \sim x^{-\alpha} \sum_{k=0}^{\infty} \frac{(-1)^{k}(\alpha)_{k}(\alpha+1-\gamma)_{k}}{k !\left(e^{-\pi i} x\right)^{k}}, \\
& \phi_{2}^{(\infty)}(x) \sim e^{x} x^{\alpha-\gamma} \sum_{k=0}^{\infty} \frac{(\gamma-\alpha)_{k}(1-\alpha)_{k}}{k ! x^{k}} .
\end{aligned}
$$

The associated monodromy matrices are as follows

$$
\begin{aligned}
& M_{0}=C(\alpha, \gamma)\left(\begin{array}{cc}
1 & 0 \\
0 & e^{-2 \pi i \gamma}
\end{array}\right) C(\alpha, \gamma)^{-1}, \quad M_{\infty}=S_{1}^{(\infty)}(\alpha, \gamma) S_{2}^{(\infty)}(\alpha, \gamma) e^{2 \pi i T_{\infty}}, \\
& e^{2 \pi i T_{\infty}}=\left(\begin{array}{cc}
e^{2 \pi i \alpha} & 0 \\
0 & e^{2 \pi i(\gamma-\alpha)}
\end{array}\right), \quad M_{0} M_{\infty}=I_{2},
\end{aligned}
$$

where $C(\alpha, \gamma), S_{1}^{(\infty)}(\alpha, \gamma)$ and $S_{2}^{(\infty)}(\alpha, \gamma)$ are connection matrix and Stokes matrices respectively which are shown in the following lemma.

Lemma 2. The Kummer's confluent hypergeometric function which is the solution of (A.2) has the following connection matrix between fundamental solutions around $x=0$ and $x=\infty$ and Stokes matrices around $x=\infty$ :

- Connection matrix:

$$
\left({ }_{1} F_{1}(\alpha, \gamma ; x) \quad x^{1-\gamma} F_{1}(\alpha+1-\gamma, 2-\gamma ; x)\right)=\left(\phi_{1}^{(\infty)}\left(e^{-\pi i} x\right) \quad \phi_{2}^{(\infty)}(x)\right) C(\alpha, \gamma),
$$

where $\phi_{i}^{(\infty)}(i \in\{1,2\})$ is the fundamental solutions around the singularity $x=\infty$ and $C(\alpha, \gamma)$ is the connection matrix which is shown as follows

$$
C(\alpha, \gamma)=\left(\begin{array}{cc}
\frac{\Gamma(\gamma) e^{\alpha \pi i}}{\Gamma(\gamma-\alpha)} & \frac{\Gamma(2-\gamma) e^{\pi i(1+\alpha-\gamma)}}{\Gamma(1-\alpha)} \\
\frac{\Gamma(\gamma)}{\Gamma(\alpha)} & \frac{\Gamma(2-\gamma)}{\Gamma(1+\alpha-\gamma)}
\end{array}\right)
$$

- Stokes matrices:

$$
S_{1}^{(\infty)}(\alpha, \gamma)=\left(\begin{array}{cc}
1 & 0 \\
\frac{-2 \pi i e^{\pi i(\gamma-2 \alpha)}}{\Gamma(\alpha) \Gamma(1+\alpha-\gamma)} & 1
\end{array}\right), \quad S_{2}^{(\infty)}(\alpha, \gamma)=\left(\begin{array}{cc}
1 & \frac{-2 \pi i e^{\pi i(4 \alpha-2 \gamma)}}{\Gamma(1-\alpha) \Gamma(\gamma-\alpha)} \\
0 & 1
\end{array}\right)
$$




\section{B Briot-Bouquet's theorem for a system of partial differential equations in two variables}

Briot and Bouquet [3] showed that existence of a holomorphic solution for a special type of nonlinear ordinary differential equations. In this section we explain the Briot-Bouquet's type theorem for a system of partial differential equations in two variables following [5].

\section{B.1 Briot-Bouquet's theorem}

Briot and Bouquet studied a nonlinear ordinary differential equation

$$
x \frac{d z}{d x}=h(z, x), \quad z=\left(z_{1}, \ldots, z_{n}\right)
$$

for $h(0,0)=0$. They have shown that if the eigenvalues of the Jacobi matrix $\left(\frac{\partial h}{\partial z}(0,0)\right)$ are not positive integers, then (B.1) has a convergent holomorphic solution.

R. Gerard and Y. Sibuya [5] studied the Briot-Bouquet's type theorem for a system of partial differential equations in two variables. They have shown that a formal solution will be convergent:

Lemma 3. Assuming that $h_{1}$ and $h_{2}$ are holomorphic functions of $z, x_{1}$ and $x_{2}$ and $z(0,0)=0$, $h_{1}(0,0,0)=h_{2}(0,0,0)=0$. If the simultaneous equations

$$
x_{1} \frac{\partial z}{\partial x_{1}}=h_{1}\left(z, x_{1}, x_{2}\right), \quad x_{2} \frac{\partial z}{\partial x_{2}}=h_{2}\left(z, x_{1}, x_{2}\right)
$$

have the formal solutions around $\left(x_{1}, x_{2}\right)=(0,0)$ expressed in power series of $x_{1}$ and $x_{2}$, they are convergent.

\section{B.2 Convergence of the solutions}

Solutions (1) and (2) are convergent by Lemma 3. For the solutions with a pole, for example, solutions (7) and (8), we put

$$
q_{1}=\frac{Q_{1}}{t_{1}}, \quad p_{1}=t_{1} P_{1}, \quad q_{2}=s_{2} Q_{2}, \quad p_{2}=\frac{P_{2}}{s_{2}},
$$

where $Q_{1}, P_{1}, Q_{2}$ and $P_{2}$ are holomorphic functions of $t_{1}$ and $s_{2}$ near $\left(t_{1}, s_{2}\right)=(0,0)$. Substituting these into the Hamiltonian system $\mathcal{H}_{2}$, it becomes all Briot-Bouquet's type differential equations with respect to $Q_{1}, P_{1}, Q_{2}$ and $P_{2}$.

\section{Acknowledgements}

The author wishes to thank Professor Y. Ohyama for his constant guidance and useful suggestions to complete this work. The author also gives thanks to the anonymous referees for their relevant contributions to improve this paper. This work was supported by JSPS KAKENHI Grant Number 22540237 and the Mitsubishi Foundation.

\section{References}

[1] Appell P., Sur les polynômes se rattachant à l'équation différentielle $y^{\prime \prime}=6 y^{2}+x$, Bull. Soc. Math. France 45 (1917), 150-153.

[2] Bolibruch A.A., On isomonodromic confluences of Fuchsian singularities, Proc. Steklov Inst. Math. 221 (1998), 117-132. 
[3] Briot C., Bouquet J.C., Recherches sur les propriétés des fonctions définies par des équations différentielles, J. de l'Ecole Polytechnique 21 (1856), 133-198.

[4] Garnier R., Étude de l'intégrale générale de l'équation VI de M. Painlevé dans le voisinage de ses singularités transcendantes, Ann. Sci. École Norm. Sup. (3) 34 (1917), 239-353.

[5] Gérard R., Sibuya Y., Étude de certains systèmes de Pfaff au voisinage d'une singularité, C. R. Acad. Sci. Paris Sér. A-B 284 (1977), A57-A60.

[6] Heading J., The Stokes phenomenon and the Whittaker function, J. London Math. Soc. 37 (1962), 195-208.

[7] Iwasaki K., Kimura H., Shimomura S., Yoshida M., From Gauss to Painlevé. A modern theory of special functions, Aspects of Mathematics, Vol. E16, Friedr. Vieweg \& Sohn, Braunschweig, 1991.

[8] Jimbo M., Monodromy problem and the boundary condition for some Painlevé equations, Publ. Res. Inst. Math. Sci. 18 (1982), 1137-1161.

[9] Kaneko K., A new solution of the fourth Painlevé equation with a solvable monodromy, Proc. Japan Acad. Ser. A Math. Sci. 81 (2005), 75-79.

[10] Kaneko K., Local expansion of Painlevé VI transcendents around a fixed singularity, J. Math. Phys. 50 (2009), 013531, 24 pages.

[11] Kaneko K., Ohyama Y., Fifth Painlevé transcendents which are analytic at the origin, Funkcial. Ekvac. 50 (2007), 187-212.

[12] Kaneko K., Ohyama Y., Meromorphic Painlevé transcendents at a fixed singularity, Math. Nachr. 286 (2013), 861-875.

[13] Kimura H., The degeneration of the two-dimensional Garnier system and the polynomial Hamiltonian structure, Ann. Mat. Pura Appl. (4) 155 (1989), 25-74.

[14] Kitaev A.V., Symmetric solutions for the first and the second Painlevé equation, J. Math. Sci. 73 (1995), 494-499.

[15] Kohno M., Global analysis in linear differential equations, Mathematics and its Applications, Vol. 471, Kluwer Academic Publishers, Dordrecht, 1999.

[16] Okamoto K., Isomonodromic deformation and Painlevé equations, and the Garnier system, J. Fac. Sci. Univ. Tokyo Sect. IA Math. 33 (1986), 575-618. 